\title{
Paciente joven en hemodiálisis con más de veinte accesos vasculares
}

\author{
Cristina Franco Valdivieso, Julia Hernando García, Mónica Brazález Tejerina, Nuria Martín Chico, Lucila \\ Fernández Arroyo, $M^{\mathrm{a}}$ Jesús Rollán de la Sota
}

Hospital Clínico Universitario. Valladolid. España

\section{Resumen}

Introducción: El acceso vascular supone un pilar fundamental en el tratamiento dialítico de los pacientes renales. El empleo de catéteres se ha incrementado en los últimos años.

Caso clínico: Varón de 49 años que inició tratamiento de hemodiálisis a sus 32 años.

Presentó 7 accesos registrados (tiempo medio de uso: 64 días) hasta el primer trasplante y otros 15 accesos (tiempo medio de uso: 162 días) y 11 infecciones por el germen Staphylococcus Epidermidis, hasta recibir el segundo trasplante, que perdió a los 5 días por infarto renal isquémico masivo, volviendo a hemodiálisis con la colocación de un catéter venoso central tunelizado femoral izquierdo, que dura hasta la actualidad sin complicaciones destacables, gracias al diagnóstico posterior de la mutación heterocigótica del factor $\mathrm{V}$ de Leiden (que provoca un trastorno de hipercoagulabilidad), comenzando anticoagulación oral con acenocumarol de forma domiciliaria y heparina de bajo peso molecular intradiálisis desde ese momento.

Discusión: Tras comenzar el sellado del catéter con Citrato + Heparina sódica (inicialmente incluía también Taurolidina pero se eliminó debido a intolerancia), no presentó más infecciones.

Debido al diagnóstico de la mutación heterocigótica del factor $V$ de Leiden, nos planteamos la posibilidad de que

Correspondencia:

Cristina Franco Valdivieso

Servicio de Nefrología

Hospital Clínico Universitario de Valladolid

C/ Ramón y Cajal, s/n. 47005. Valladolid

E-mail: cfranco@saludcastillayleon.es los fracasos de los accesos anteriores sean debidos al desconocimiento de esta mutación.

Ante la situación demográfica de España se pone de manifiesto la necesidad de equipos multidisciplinares más amplios e incluir un control y seguimiento del acceso tunelizado para reducir sus pérdidas y evitar situaciones altamente invasivas.

PALABRAS CLAVE: acceso vascular; hemodiálisis; catéter venoso central; factor $\mathrm{V}$.

\section{Young patient on hemodialysis with more than twenty vascular access}

\section{Abstract}

Introduction: The vascular access is a basic pillar in the dialytic treatment of renal patients. The use of catheters has increased in recent years.

Clinical case: 49 year old male who started hemodialysis treatment at 32 years. He presented 7 registered access (average use time: 64 days) until the first transplant and other 15 accesses (average use time: 162 days) and 11 infections by Staphylococcus Epidermidis, until receive the second transplant. He lost the graft 5 days after by massive renal ischemic infarction, returning to hemodialysis with the placement of a central venous catheter tunneled in left femoral, that last to the present day without notable complications, mainly due to the subsequent diagnosis of heterozygous mutation in the factorV Leiden (that causes a hypercoagulability disorder), starting oral anticoagulation with acenocoumarol in his home and intradialytic heparin low molecular weight since that time. 
Discussion: After starting the sealed catheter with citrate + heparin sodium (initially it also includes Taurolidina, but it was removed due to intolerance), did not give more infections.

Because of the diagnosis of heterozygous factor V Leiden mutation, we consider the possibility that the previous access failures are due to ignorance of this mutation.

Given the demographic situation in Spain, we highlight the need to expand the multidisciplinary teams and include a protocol of control and monitoring of the tunneled access to reduce their losses and avoid a highly invasive.

KEYWORDS: vascular access; hemodialysis; central venous catheter; factor $\mathrm{V}$.

\section{Introducción}

El acceso vascular (AV) supone un pilar fundamental en el tratamiento dialítico de los pacientes renales ${ }^{1}$. A pesar de estar establecida la fístula arteriovenosa interna (FAV) autóloga como primera opción y la fístula arteriovenosa protésica con injerto de politetrafluoroetileno (PTFE) en segundo lugar como AV idóneos según las "Guías españolas de Accesos Vasculares $^{\prime 2}$, el empleo de catéteres tanto no tunelizados como tunelizados se ha incrementado en los últimos $a_{n}{ }^{3}{ }^{3}$. Normalmente esta situación ocurre en pacientes añosos ${ }^{4}$ que tienen un mayor deterioro de la red vascular, lo que les influye negativamente en el desarrollo del acceso, pero a veces este problema se da también en personas inesperadas, como es el caso del paciente que nos ocupa.

Se debe destacar que tener un acceso venoso central, tanto tunelizado como no tunelizado, supone un riesgo potencial de complicaciones y mortalidad, siendo el de tipo infeccioso el que más morbimortalidad presenta ${ }^{5}$, además de problemas relacionados con el funcionamiento del catéter o deterioro estructural del mismo que supone en ocasiones su recambio6.

\section{Caso Clínico}

Varón de 49 años que inicia tratamiento de hemodiálisis (HD) desde agosto de 2000 (a la edad de 32 años) por insuficiencia renal crónica secundaria, debida posiblemente a pielonefritis crónica. Seguido en consulta de nefrología y urología desde 1988.
ANTECEDENTES PERSONALES: sin alergias medicamentosas conocidas (con intolerancia a Carbonato Cálcico y Taurolidina). Exfumador y exbebedor.

ANTECEDENTES MÉDICOS: gota úrica desde 1995, rectorragia en enero 2009, hipertensión arterial en tratamiento con una crisis hipertensiva en 2012, hipocalcemia moderada-severa sintomática postparatiroidectomía desde 2013, EPOC moderado desde abril de 2014, cuadro de intoxicación por monóxido de carbono en noviembre de 2014, politransfundido e hiperinmunizado, hipotiroidismo subclínico, bronconeumonía crónica obstructiva, plaquetopenia, temblor esencial familiar, hiperpotasemias e hiperfosfatemias severas secundarias a transgresiones dietéticas.

INTERVENCIONES QUIRÚRGICAS: trasplante renal de cadáver en noviembre de 2001 con pérdida del injerto en 2008 por nefropatía crónica del injerto, reiniciando hemodiálisis periódica en septiembre de 2008. Paratiroidectomía subtotal (se conserva 1/3 de paratiroides derecho) + hemitiroidectomía izquierda en marzo de 2013. Trasplante renal de cadáver en abril de 2015 con pérdida por infarto renal masivo por trombosis a los 5 días y vuelta a HD periódica. Canalización de numerosos accesos vasculares (AV) (Tabla 1), con cuadros de infecciones secundarias a ellos.

HISTORIA DE ACCESOS VASCULARES: durante el periodo comprendido entre el inicio de la enfermedad renal en el año 2000 y el primer trasplante en el año 2001, tuvo 7 AV registrados: 2 catéteres yugulares temporales, 2 intentos de FAVI, 2 PTFE y por último 1 catéter permanente. El tiempo medio de uso de estos accesos fue de 64 días, siendo el mínimo uso 0 días (FAV no funcionante) y el máximo de 137 días (PTFE). En este intervalo de tiempo no se registraron infecciones relacionadas con los accesos.

Reinició tratamiento sustitutivo renal tras el fracaso del primer trasplante en septiembre de 2008 por nefropatía crónica del injerto. En este periodo tuvo 15 AV: 1 intento de FAV, 4 catéteres temporales y 10 permanentes. EI tiempo medio de uso fue de 162 días, siendo el mínimo uso 0 días (FAV no funcionante) y el máximo de 664 días (catéter permanente femoral derecho). En este intervalo de tiempo se encontraron 11 infecciones registradas, en algunos casos con extrusión del manguito, siempre por el germen Staphylococcus Epidermidis, nunca por el germen habitual en estos pacientes (Staphylococcus Aureus). 
Table 1. Historial de Accesos Vasculares.

\begin{tabular}{|c|c|c|}
\hline FECHA & ACCESO VASCULAR & DÍAS DE USO \\
\hline 20/08/2000 & CVNT yugular derecho & 105 \\
\hline 05/09/2000 & FAVn radio-cefálica derecha (no funcionante) & 0 \\
\hline $12 / 09 / 2000$ & FAVn radio-cefálica derecha (no funcionante) & 0 \\
\hline 03/11/2000 & FAVp brazo izquierdo & 167 \\
\hline 19/05/2001 & CVNT yugular derecho & 87 \\
\hline 26/07/2001 & FAVp brazo izquierdo & 0 \\
\hline 14/08/2001 & CVT yugular derecho & 92 \\
\hline 03/09/2008 & CVNT yugular izquierdo & 73 \\
\hline 15/11/2008 & CVNT yugular izquierdo & 55 \\
\hline 09/01/2009 & CVNT subclavio derecho & 88 \\
\hline 09/02/2009 & FAVn húmero-cefálica dcha (no funcionante) & 0 \\
\hline 07/04/2009 & CVT subclavio derecho & 73 \\
\hline 19/06/2009 & CVNT femoral derecho & 11 \\
\hline 30/06/2009 & CVT femoral derecho & 273 \\
\hline 30/03/2010 & CVT femoral derecho & 664 \\
\hline 23/01/2012 & CVT femoral derecho & 42 \\
\hline 05/03/2012 & CVT femoral izquierdo & 238 \\
\hline 29/10/2012 & CVT femoral izquierdo & 518 \\
\hline $31 / 03 / 2014$ & CVT femoral izquierdo & 52 \\
\hline $22 / 05 / 2014$ & CVT femoral izquierdo & 250 \\
\hline $27 / 01 / 2015$ & CVT femoral izquierdo & 49 \\
\hline $17 / 03 / 2015$ & CVT femoral izquierdo & 41 \\
\hline $27 / 04 / 2015$ & CVT femoral izquierdo & 891 \\
\hline
\end{tabular}

CVNT: Catéter venoso central no tunelizado. CVT: Catéter venoso central tunelizado. FAV: Fístula arterio venosa nativa. FAVp: Fístula arteriovenosa protésica.

En 2014, se modificó el sellado habitual del catéter por una presentación farmacéutica que incluye Taurolidina + Citrato + Heparina sódica. Tras presentar una reacción alérgica se modificó a un sellado con Citrato + Heparina sódica.

Se sometió a un nuevo trasplante renal de cadáver en abril de 2015 con pérdida por infarto renal isquémico masivo por trombosis a los 5 días volviendo a HD periódica con colocación de un nuevo AV permanente femoral izquierdo que dura hasta la actualidad. A pesar de ser un catéter permanente normofuncionante, la dosis de diálisis media conseguida es insuficiente $(\mathrm{KT} / \mathrm{V}=0,8-0,9)$. Se modificó la técnica dialítica, el tamaño del dializador y además se propuso un aumento del tiempo de diálisis al paciente, el cual rechazó.

Tras la trombosis del segundo trasplante en 2015, se diagnosticó la mutación heterocigótica del factor $\mathrm{V}$ de Leiden (que provoca un trastorno de hipercoagulabili- dad), comenzando anticoagulación oral con Acenocumarol de forma domiciliaria y heparina de bajo peso molecular intradiálisis desde ese momento.

Con el diagnóstico de la mutación y su tratamiento, el paciente decide continuar incluido en lista de trasplante renal, a pesar de los riesgos existentes de la técnica, ya que la intervención de un nuevo trasplante supondrá la retirada del acceso vascular in situ.

Actualmente el paciente lleva dos años y medio con un catéter permanente femoral, sin complicaciones infecciosas ni de funcionamiento destacables.

\section{Discusión}

Hasta 2014, el paciente sufría numerosas infecciones con extrusión del manguito, siempre por el germen Staphylococcus Epidermidis. Destaca la desaparición de las infecciones con la introducción del sellado del catéter con la presentación que incluía Taurolidina + Citrato + Heparina sódica. Debido a la reacción alérgica, se modificó dicho sellado a otro con Citrato + Heparina sódica, manifestándose que es igual de efectivo en el tratamiento preventivo de infecciones en pacientes con intolerancia 0 alergia a Taurolidina.

Debido al diagnóstico de la mutación heterocigótica del factor $\mathrm{V}$ de Leiden (que provoca un trastorno de hipercoagulabilidad), nos planteamos la posibilidad de que los fracasos de los AV anteriores sean debidos al desconocimiento de esta mutación.

Tanto Rodríguez Hernández et al. ${ }^{7}$ como Roca ${ }^{8}$, coinciden en que es necesaria la implantación de programas de monitorización del AV con un equipo multidisciplinar que incluyen a nefrología, enfermería, radiología y cirugía vascular, teniendo como objetivo principal la supervivencia del acceso, aunque estos programas ponen de protagonista a la FAV, ya sea nativa o protésica.

Ante la situación demográfica de España, con un envejecimiento de la población y la existencia de comorbilidades asociadas a ello, que dificultan el correcto desarrollo 
de una FAV, este caso pone de manifiesto la necesidad de crear equipos multidisciplinares más amplios en especialidades médicas y una revisión para incluir el control y seguimiento del AV tunelizado de los pacientes en HD para reducir las pérdidas de estos AV y evitar situaciones altamente invasivas.

El autor declara que no hay conflicto de interés.

Recibido: 30 noviembre 2017

Revisado: 15 enero 2018

Modificado: 28 enero 2018

Aceptado: 30 enero 2018
5. Fortún J. Infecciones asociadas a dispositivos intravasculares utilizados para la terapia de infusión. Enferm Infecc Microbiol Clin 2008;3(26):168-74.

6. Alarcia Martín A, Brazález Tejerina M, Franco Valdivieso C, Melero Salas L, Rollán de la Sota, MJ. Reparación de catéteres tunelizados de hemodiálisis con moldes de silicona. Enferm Nefrol 2013;4(16): 281-83.

7. Rodríguez Hernández JA, González Parra E, Gutiérrez Julián JM, Segarra Medrano A, Almirante B, Martínez MT et al. Vigilancia y seguimiento del acceso vascular. Nefrología 2005;25(1):34-47.

8. Roca R. Control de los accesos vasculares. Exploración física e instrumental. En: González Álvarez MT, Martínez Cercós R. Manual de accesos vasculares para hemodiálisis. Madrid: Marge Médica Books; 2010. p. 87-97.

\section{Bibliografía}

1. Schwab S, Besarab A, Beathard G, Brouwer D, Levine M, McCann R, et al. III. NKF-K/DOQI Clinical Practice Guidelines for Vascular Access: update 2000. Am J Kidney Dis. 2001 Jan;37(1 Supl 1):S137-81.

2. Barbosa F. Indicación del acceso vascular desde el punto de vista nefrológico. En: González Álvarez MT, Martínez Cercós R. Manual de accesos vasculares para hemodiálisis. Madrid: Marge Médica Books; 2010. p. 25-35.

3. Roca Tey R. El acceso vascular para hemodiálisis: la asignatura pendiente. Nefrología 2010; 3 (30): 280-7.

4. López Gómez JM. Avances en hemodiálisis clínica: accesos vasculares y pacientes de edad avanzada. Nefrología 2008; 28(5):67-70.

Este artículo se distribuye bajo una Licencia Creative Commons Atribución-NoComercial 4.0 Internacional. https://creativecommons.org/licenses/by-nc/4.0/

\section{Open Access (c) (i) \&}

